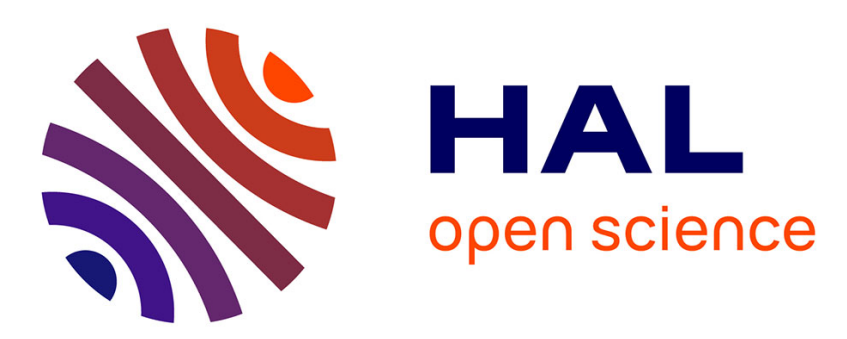

\title{
Angular dependence of the tunnel magnetoresistance in transition-metal-based junctions
}

H. Jaffrès, D Lacour, F. Nguyen van Dau, J. Briatico, F. Petroff, A. Vaurès

\section{To cite this version:}

H. Jaffrès, D Lacour, F. Nguyen van Dau, J. Briatico, F. Petroff, et al.. Angular dependence of the tunnel magnetoresistance in transition-metal-based junctions. Physical Review B: Condensed Matter (1978-1997), 2001, 64 (6), pp.064427. 10.1103/PhysRevB.64.064427 . hal-02954107

\section{HAL Id: hal-02954107 \\ https://hal.science/hal-02954107}

Submitted on 30 Sep 2020

HAL is a multi-disciplinary open access archive for the deposit and dissemination of scientific research documents, whether they are published or not. The documents may come from teaching and research institutions in France or abroad, or from public or private research centers.
L'archive ouverte pluridisciplinaire HAL, est destinée au dépôt et à la diffusion de documents scientifiques de niveau recherche, publiés ou non, émanant des établissements d'enseignement et de recherche français ou étrangers, des laboratoires publics ou privés. 


\title{
Angular dependence of the tunnel magnetoresistance in transition-metal-based junctions
}

\author{
H. Jaffrès, D. Lacour, F. Nguyen Van Dau, J. Briatico, F. Petroff, and A. Vaurès \\ Unité Mixte de Physique CNRS/Thomson-CSF, Domaine de Corbeville, Orsay, France \\ (Received 27 November 2000; revised manuscript received 2 March 2001; published 24 July 2001)
}

\begin{abstract}
We have investigated the angular behavior of the tunnel magnetoresistance (TMR) in transition-metal-based junctions using the low-field susceptibility of the crossed magnetic configuration. The noncollinear arrangement, stabilized by combining step anisotropy and interfacial exchange-bias coupling, is shown to be of a particular interest for an accurate analysis of the angular dependence of the TMR. We show that the intrinsic tunnel processes are reflected on a linear behavior of the conductivity giving a more complex form for the resistance, as expected by the model of Slonczewski. The more intuitive "high-field" saturating regime deviates the hard layer from its nominal pinning direction and consequently is shown to be less adapted for the experimental study of the intrinsic angular response of the TMR.
\end{abstract}

DOI: 10.1103/PhysRevB.64.064427

PACS number(s): 75.30.Gw, 73.40.Gk, 73.40.Rw

\section{INTRODUCTION}

Since the work reported by Moodera et al. ${ }^{1}$ and Miyazaki and Tezuka, ${ }^{2}$ the physics of magnetic-tunnel junctions (MTJ's) have attracted much attention, partly explained by the will of a better understanding of the fundamental processes of tunneling through a thin insulating solid film.

The magnetoresistance of tunnel junctions ${ }^{3}$ (TMR), i.e., the normalized difference of their conductivity measured in the parallel $\left(\mathrm{G}_{p}\right)$ and antiparallel $\left(\mathrm{G}_{a p}\right)$ magnetic configurations, results in the spin polarization of the ferromagnetic (FM) electrodes at the Fermi level. ${ }^{4}$ In magnetic junctions, one observes generally an abrupt transition between the two states when the external magnetic field just overcomes the coercivity of one electrode. Nevertheless, up to now, no large studies have been reported on the variation of the MTJ conductivity occurring when the relative orientation between the two magnetization vectors varies continuously. Evidencing this TMR angular dependence would be particularly interesting from a technological point of view if one has in mind to realize high controllable and reliable magnetic sensors ${ }^{5-8}$ or read head ${ }^{9-11}$ supplying a large signal sensitivity, linearity, and reversibility.

Contrary to the giant-magnetoresistance (GMR) effects described by pure diffusive processes at interfaces, ${ }^{12,13}$ the tunnel current has to reflect a certain quantum probability $t_{\uparrow \uparrow}=t_{\downarrow \downarrow}=T_{\uparrow \uparrow} \cos ^{2}(\theta / 2)$ for a spin-up (-down) electron of one electrode to be transferred into a spin-up (-down) state of the second electrode when the two quantization axes within each side of the barrier make an angle $\theta \cdot{ }^{14}$ Through the very general Landauer formula, ${ }^{15,16}$ the conductivity may be viewed as the macroscopic physical observable of the transmission coefficients. Consequently, neglecting spin-flip effects, i.e., the $t_{\uparrow \downarrow}$ terms, the existence of two spin channels has to be reflected on a linear angular variation of the tunnel conductivity vs $\cos \theta$,

$$
\begin{aligned}
G & =G_{p} \cos ^{2} \frac{\theta}{2}+G_{a p} \sin ^{2} \frac{\theta}{2} \\
& =\bar{G}\left(1+\frac{\mathrm{TMR}}{2} \cos \theta\right)
\end{aligned}
$$

leading to a more complex form for the resistance,

$$
R=\frac{1}{G}=\frac{R_{\perp}}{1+\frac{\mathrm{TMR}}{2} \cos \theta} .
$$

This differs ${ }^{17}$ from what is proposed generally ${ }^{18}$ giving a linear variation of the resistance vs. $\cos \Theta$ that appears correct only for small absolute values of tunnel magnetoresistance.

This property would be evidenced by measuring the resistance of "analog" tunnel spin valve wherein the opening angle $\Theta$ is controlled by an external magnetic field. Ideally, this requires the preparation of junctions constituted of a very sensitive electrode, a macrospin with a high susceptibility able to describe the film plane, i.e., free of internal forces (no anisotropy) as well as local magnetic domains, source of irreversible processes. In addition, the counterelectrode has to be formed by a "hard" layer with its magnetization locked along a reference direction. All these conditions are hardly fulfilled together and this explains why interpretation of the angular response beyond the simple cosine shape is not trivial, because it includes undesirable effects intrinsic to the material properties.

However, once the magnetic properties and consequently the response of the magnetic layers are perfectly under control, which is generally possible in the low-field range, interpretations of angular variations may become clearer. In this spirit, the high susceptibility supplied by the perpendicular magnetic arrangement is of a particular interest. This constitutes the central point of this work. The method we used consists in controlling the direction of the softer magnetization away from its easy axis in the coherent-rotation regime keeping fixed the "hard" countermagnet. This is made possible by taking advantage of the shape effects introduced by vicinal step-bunched $\mathrm{Si}(111)$ substrates inducing a uniaxial anisotropy. ${ }^{19}$

To our knowledge, this is the first detailed angular study of the tunnel magnetoresistance that allows us to address the 
question of validity of Eq. (2) and, in addition, the role of the anisotropic character of the magnetic films prepared for tunnel devices.

The paper is organized as follows. We first present the sample preparation together with the method of crossing the two magnetization vectors beyond and below the barrier (Sec. II). In Sec. III (IV), we present a detailed study of the low- (high-) field angular response combining experimental measurements and models based on the coherent magnetization reversal. A particular emphasis will be placed on the advantages of the low-field measurements that are shown to be the most valuable method to extract the real and intrinsic angular dependence of the tunnel conductivity.

\section{JUNCTION PREPARATION}

Crossing anisotropies is aimed at the realization of linear magnetic sensors. ${ }^{6,7}$ This requires a sensitive magnetic film interacting linearly with the field to be detected and a hard magnet, used as an analyzer, giving the sensitive direction. ${ }^{20}$ The noncollinear configuration may be stabilized through magnetic anisotropies induced independently in each electrode by different methods combining artificial shape anisotropy, ${ }^{5,21}$ unidirectional exchange bias, ${ }^{6,7,10,11}$ or artificial antiferromagnetic coupling $22,23(\mathrm{AAF})$, for instance. We have taken advantage of the step energy to induce a welldefined easy axis within the bottom electrode grown on a step-bunched $\mathrm{Si}(111)$ substrate. The easy axis coincides with the large dimension of the terraces appearing during the socalled step-bunching process occurring during a thermal treatment carried out on a misoriented $\mathrm{Si}(111)$ substrate. ${ }^{19}$ Previous magnetic studies ${ }^{24}$ have clearly shown that the stepbunched mechanism promotes well-defined anisotropic direction and strength $H_{K}^{\text {Step }}$ over several centimeters. The surface modulation is obtained by thermal treatment of a nominal $\mathrm{Si}(111)$ substrate $4^{\circ}$ or $8^{\circ}$ misoriented towards the $[11 \overline{2}]$ direction. Details of the step-bunching mechanism are given elsewhere. ${ }^{19,25}$ The perpendicular arrangement is then obtained by using the unidirectional exchange anisotropy generated in a FM layer by an antiferromagnetic (AF) material grown beyond ${ }^{26-28}$ as it was done in the spin-valve GMR structure. ${ }^{29}$ In the present case, the AF $\mathrm{CoO}$ compound was chosen for its intrinsic large exchange strength. ${ }^{30}$ The procedure consists then in cooling down the structure in a saturating magnetic field applied perpendicularly to the steps in the vicinity of the Néel temperature $\mathcal{T}_{\mathcal{N}} \simeq 180 \mathrm{~K}$ of thin $\mathrm{CoO}$ films. ${ }^{30}$

The $\mathrm{Co} / \mathrm{Al}_{2} \mathrm{O}_{3} / \mathrm{Co} / \mathrm{CoO} / \mathrm{Au}$ junctions were grown in a commercial Alcatel sputtering system. A 15-nm-thick Co electrode is first deposited by radio frequency (rf) sputtering on Si wafers in a 4-m Torr argon plasma. A tunnel barrier is then formed by deposition of a 1.5 -nm-thick aluminum layer oxidized in a 2-m Torr $\mathrm{Ar}_{2} / 2-\mathrm{m}$ Torr $\mathrm{O}_{2}$ rf plasma for $1 \mathrm{~min}$. A 15-nm Co counterelectrode is then deposited in the same plasma conditions as the bottom one. An AF thin $\mathrm{CoO}$ film is synthesized beyond the top electrode by oxidization of the Co layer in a 2-m Torr $\mathrm{Ar}_{2} / 2-\mathrm{m}$ Torr $\mathrm{O}_{2}$ rf plasma for $1 \mathrm{~min}$. A $15-\mathrm{nm}$ Au cap layer is finally deposited to prevent any

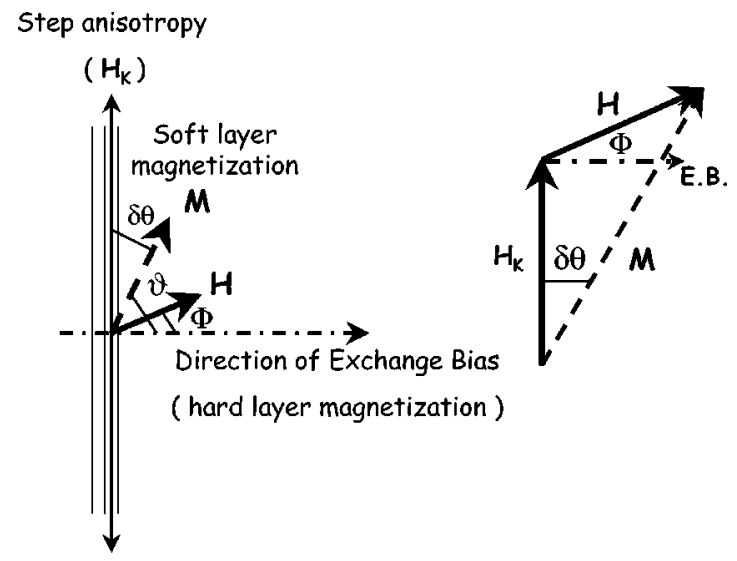

FIG. 1. Principle of low-field angular resistance measurement. The softer electrode is forced to turn coherently by an angle $\delta \theta$ towards its hard direction by the external field. The hard magnet is pinned along the exchange-bias (EB) direction. The soft magnetization aligns along the effective field $H_{K}+H$.

overoxidization. The $\mathrm{CoO}$ layer thickness is estimated to about $1.5 \mathrm{~nm}$.

For magnetotransport measurements, tunnel junctions are patterned in four steps using optical lithography processes. Circle-shaped junctions with dimensions ranging from 10 to $300 \mu \mathrm{m}$ are patterned with a first milling step stopped in the bottom electrode. A second milling step allows us to pattern a stripe-shaped $400-\mu \mathrm{m}$-wide bottom contact. Finally, insulator and metal pads are deposited in order to take contacts on each electrode. ${ }^{31}$ The intrinsic resistance of the tunnel junctions was measured to be equal to $2.5 \Omega \mathrm{cm}^{2}$ at low bias $(10 \mathrm{mV})$ and at low temperature $(30 \mathrm{~K})$. This corresponds to a barrier thickness of $1.5 \mathrm{~nm}$ and a barrier height of $1.05 \mathrm{eV}$. These latter values were taken from the Brinckman et al. fit $^{32}$ realized on $I(V)$ curves measured for a demagnetized state. The bias dependence of the magnetoresistance (not reported here) shows a classical behavior with a maximal TMR equal to $25 \%$ and a $V_{1 / 2}$ equal to $0.32 \mathrm{~V}$ at which the magnetoresistance is reduced by a factor 2 . All measurements presented below have been performed at $T=30 \mathrm{~K}$.

\section{LOW-FIELD ANGULAR BEHAVIOR}

\section{A. Experiments}

In this first part, we present the low-field angular behavior of the resistance. This corresponds to magnitude $H$ smaller than both the switching and anisotropy fields of the bottom electrode (respectively, $H_{C}=50$ Oe and $H_{K}^{\text {Soft }}=100 \mathrm{Oe}$ ). The external field $H$, oriented at an angle $\Phi$ counted from the reference exchange-bias direction, forces a slight rotation of the softer magnetization away from its equilibrium position. This induces, in parallel, a small modulation $\delta \theta$ of the relative orientation between the two magnets. This is pictured in Fig. 1.

The Fig. 2(a) displays the angular response measured for $H=10$ Oe. The normalization has been performed from the state $R_{\perp}$ corresponding to the perpendicular arrangement stabilized at zero field. This explains the respective positive and negative sign of the signal depending on the angle $\Phi$. 

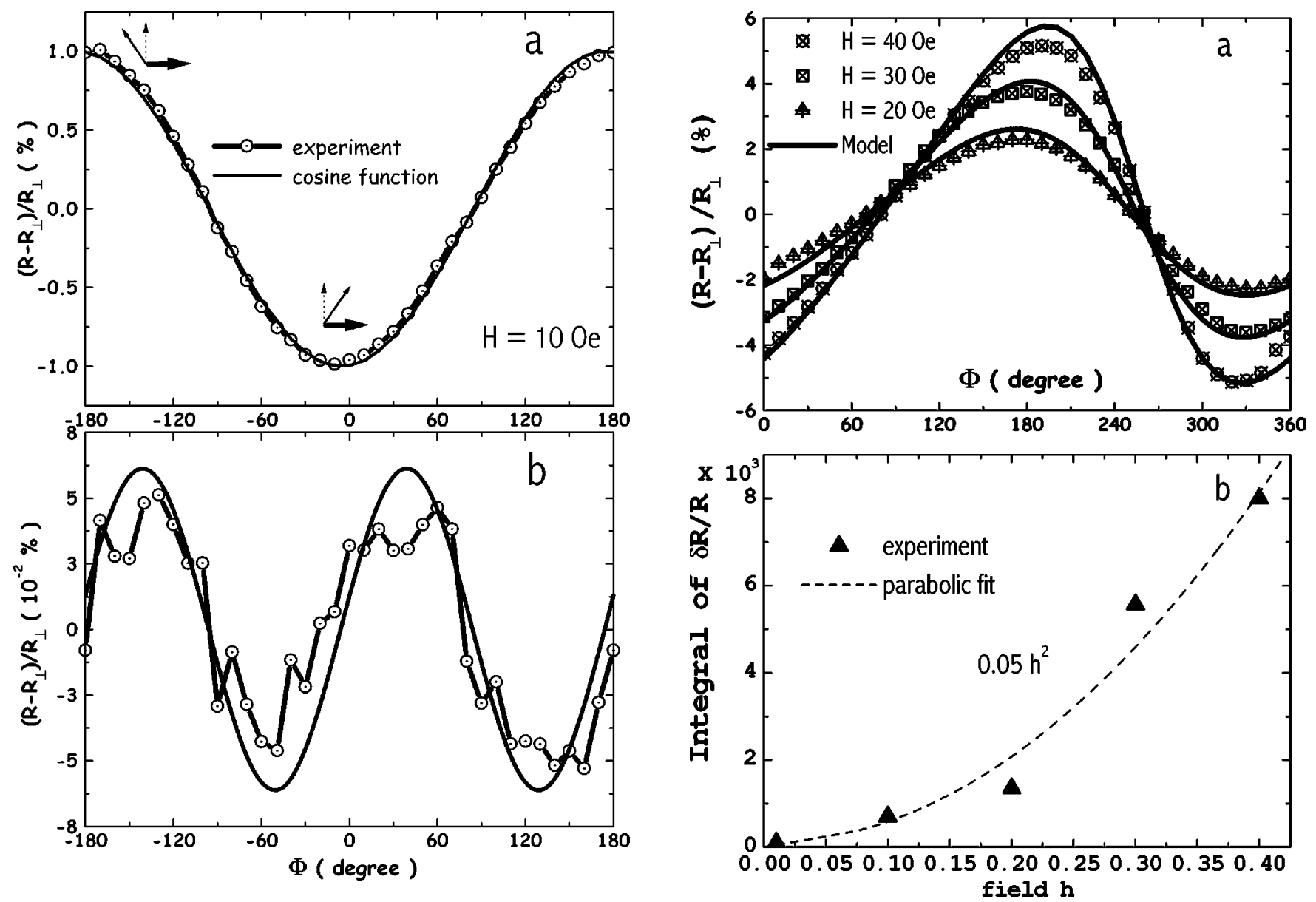

FIG. 2. (a) Angular dependence of the resistance measured for $H$ equal to $10 \mathrm{Oe}$. The arrows indicate the magnetic arrangement (thick arrow, hard magnet; thin arrow, soft magnet; dash arrow, nominal position at $H=0$ ). The signal, which was measured to be reversible, presents a quasicosine shape (straight line). (b) The deviation of the angular TMR response from a $-\cos (\Phi+\phi)$ function appears like quadratic effects of double frequency. The straight line reproduces the simple model described in Sec. III B.

The response was measured to be reversible, which emphasizes the coherent nature of the magnetization reversal as expected for this range of field. The sinelike shape of the resistance variation vs $\Phi$ with a peak-to-peak magnitude of $2 \%$ does not foresee the same intrinsic angular dependence on $\theta$ because of the complex relationship linking $\theta$ and $\Phi$. This point will be addressed in the following. The minimum (maximum) of the resistance is obtained for about $\Phi=0$ $(\Phi=\pi)$, which expresses a decrease (increase) in the relative angle between the two magnetizations. Figure 2 shows that the output resistance can be well reproduced by a cosine function of the form $\delta R^{(1)}(\Phi)=\Delta R \cos (\Phi-\pi+\phi)$. $\phi$ is a small phase shift that is induced by the presence of the interlayer coupling field $H_{d}$ acting on the softer one as a small bias. ${ }^{33}$ Because of this small phase shift, the magnetic configuration at zero field slightly deviates from the perpendicular configuration $\left(\theta_{0}=\pi / 2-\phi\right.$ instead of $\left.\pi / 2\right)$. Its value was evaluated through the following relationship:

FIG. 3. (a) Angular dependence of the resistance measured for $H=20,30$, and 40 Oe (symbols) together with expected responses (straight lines) obtained from formula (12). (b) Dependence on $h$ of the circular integral of $\delta R / R_{\perp}$. It presents a quasiquadratic dependence on $h$ with a curvature equaling 0.05 .

$$
\sin \phi=\frac{1}{\pi \Delta R} \int_{0}^{2 \pi} R(\Phi) \sin \Phi d \Phi
$$

and was found to be equal to $\phi \simeq 5^{\circ}$.

The deviation from an ideal cosine function, displayed in Fig. 2(b), clearly points out a higher harmonic of the form $\delta R^{(2)}\left(H^{2}\right) \sin 2 \Phi$. With a simple argument, one can say that this reflects a quadratic contribution in $H$. Indeed, the identity transformation $H \rightarrow-H$ and $\Phi \rightarrow \Phi+\pi$ proves that $\delta R^{(2)}\left(H^{2}\right)$ must be even on $H$.

Increasing the field magnitude to $H=20,30$, and $40 \mathrm{Oe}$, induces a larger peak-to-peak response, respectively, $4.6 \%$, $7.3 \%$, and $10.3 \%$ quasilinearly linked to $\mathrm{H}$ [Fig. 3(a)]. We can also note a progressive distortion from the cosine shape related to the occurrence of higher-order terms $\delta R^{(n)}$. Following the arguments discussed in the beginning of the section, distortions have two sources: the intrinsic variation of the tunnel response, together with a complex form of the magnetic susceptibility in the crossed-bias geometry.

The next section is devoted to the discussion of the intrinsic angular dependence of the resistance taking into account 
the ability of the sensitive layer to be uniformly magnetized along the step anisotropy field.

\section{B. Model}

\section{Magnetic susceptibility within the crossed geometry}

Until the external magnetic field overcomes the switching field $H_{C}$, the sensitive layer behaves like a macrospin that experiences both the step anisotropy field $H_{K}^{\text {Step }}$ and the external field $H$ (see Fig. 1). The equilibrium position is given by

$$
\sin \delta \theta \cong \delta \theta=-\frac{H \cos \Phi}{H_{K}+H \sin \Phi} .
$$

If we introduce the reduced field $h=H / H_{K}^{\text {Step }}$ and a phase shift $\phi$ linked to the presence of a dipolar field $\sin \phi$ $=H_{d} / H_{K}^{\text {Step }}$, the angle deviation $\delta \theta$ is expressed as ${ }^{34}$

$$
\delta \theta=-\frac{h \cos (\Phi+\phi)}{1+h \sin (\Phi+\phi)},
$$

which is correct when $h$ is small compared to unity.

The general expression (5) giving the real-angle modulation breaks up respectively into a linear $\delta \Theta^{(1)}$ and a quadratic $\delta \Theta^{(2)}$ response like

$$
\delta \Theta^{(1)}=-h \cos (\Phi+\phi)
$$

and

$$
\delta \Theta^{(2)}=h^{2} \cos (\Phi+\phi) \sin (\Phi+\phi)=\frac{h^{2}}{2} \sin [2(\Phi+\phi)],
$$

which describes the angular behavior of the resistance observed experimentally.

\section{Linear response of the resistance}

Starting from the generic equation (1), the change of the conductivity $\delta \mathrm{G}$, at first order, appears to be proportional to $\sin \Theta_{0} \delta \Theta$. The relative variation of the resistance is then linked to the angle modulation $\delta \Theta$ according to

$$
\frac{\delta R^{(1)}}{R} \simeq \frac{-\delta G}{G}=\frac{1}{2} \frac{G_{p}-G_{a p}}{\bar{G}} \sin \Theta_{0} \delta \Theta
$$

leading to the following variation of the resistance:

$$
\frac{\delta R}{R_{\perp}} \simeq \frac{\mathrm{TMR}}{2} h \cos (\Phi+\phi) .
$$

At very low field $(h \ll 1)$, the peak-to-peak signal magnitude is equal to TMR $\times h$, i.e., $0.25 \% / \mathrm{Oe}$ in the present case. This is in agreement with experiments indicating a $2 \%$ relative variation for $10 \mathrm{Oe}$ (Fig. 2). The difference may originate from the error made on the field value of about \pm 1 Oe.

If we focus on the phase, the reduced coupling field $H_{d} / H_{K}$ is equal to $\sin \phi$ giving

$$
H_{d}=H_{K} \sin \phi,
$$

i.e., about 8 Oe in agreement with the value deduced from the $R(H)$ minor loop.

\section{Quadratic response of the resistance}

Merging Eqs. (1) or (2) together with Eq. (7) gives the quadratic response of the resistance,

$$
\frac{\delta R^{(2)}}{R_{\perp}} \simeq \frac{\mathrm{TMR}}{4} h^{2} \sin 2(\Phi+\phi)
$$

corresponding to the upper harmonic $\sin 2(\Phi+\phi)$ with a peak-to-peak magnitude equal to $(\mathrm{TMR} / 2) h^{2}$. This is in perfect agreement with the experimental curve [Fig. 2(b)].

The evidence of quadratic effects shows that, if one considers the linear response $\delta R^{(1)}$, the error made on the angle $\Phi$, given by $\Delta \Phi=\delta R^{(2)} /\left(\partial \delta R^{(1)} / \partial \Phi\right)=-h \cos \Phi$, is scaled down by the anisotropy constant $H_{K}$. This means that a gain of sensitivity would lead to a loss of angular precision, and inversely.

\section{Higher order in $h$}

We now will focus on the question addressed in the Introduction about the real angular dependence of the tunnel magnetoresistance, i.e., if the forms (1) and (2) are fully correct.

Considering the magnetic response $\delta \theta$ given by Eq. (5), the resistance is expected to vary with the field like

$$
\frac{\delta R}{R_{\perp}} \simeq-\frac{\frac{\mathrm{TMR}}{2} h \cos (\Phi+\phi)}{1+h \sin (\Phi+\phi)+\frac{\mathrm{TMR}}{2} h \cos (\Phi+\phi)} .
$$

This function reproduces experimental data faithfully [Fig. 3 (b)] without any use of floating parameters. The numerator of Eq. (12) represents the linear dependence on $h$ as expressed by Eq. (9). The quadratic part is also recovered [Eq. (11)] considering an absolute value of $R_{T}$ small compared to 1 . The nonlinear angular dependence of the resistance as claimed by Eq. (2), is related to the term $(\mathrm{TMR} / 2) h \cos (\Phi+\phi)$ appearing in the denominator of Eq. (12). This introduces a phase shift ${ }^{35}$ that can be evaluated by performing the circular integral of $\delta R / R_{\perp}$ (Ref. 36),

$$
\int_{0}^{2 \pi} \frac{\delta R}{R_{\perp}} d \Phi \simeq \frac{\pi}{8}(\mathrm{TMR})^{2} h^{2} .
$$

In Fig. 3(b), we have reported the experimental integral values $I(h)$ corresponding to $h=0,0.1,0.2,0.3$, and 0.4 . It is quite remarkable to note a quadratic shape for $I(h)$ close to the expected one with a curvature 0.05 approaching $\pi / 8\left[(\mathrm{TMR})^{2}\right]=0.03$ from formula (13). Both the shift and the integral vanish if one considers a linear dependence of the resistance on $\cos \theta \cdot{ }^{35}$ In that sense, the integral treatment constitutes the main powerful argument pointing out the validity of the expression (12) related to a linear response of the conductivity vs $\cos \theta$.

We will focus now on the angular response performed at higher fields. It corresponds to the case whereby the field $H$ 


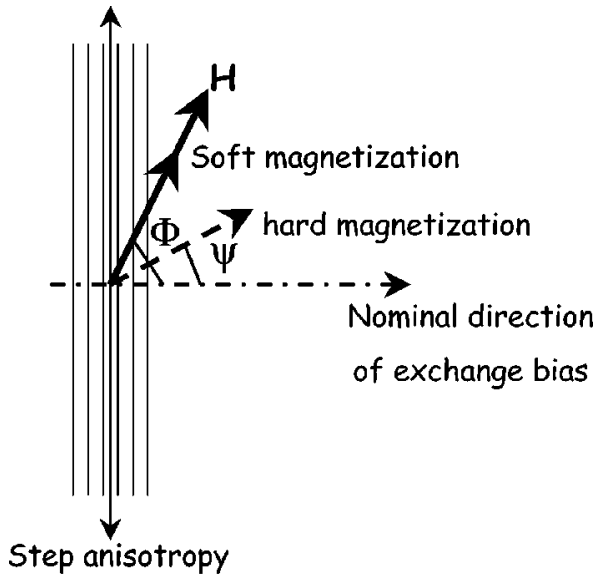

$\left(H_{k}\right)$

FIG. 4. Principle of high-field angular detection. The softer electrode is forced to be oriented along the field whereas the hard magnet is pinned along the exchange-bias direction. $\psi$ takes into account a slight deviation from this nominal direction.

is larger than the step anisotropy $H_{K}^{\text {Step }}$, saturating the softer electrode, but remains much smaller than the unidirectional and/or uniaxial exchange bias.

\section{HIGH-FIELD ANGULAR BEHAVIOR}

\section{A. Experiment}

In this case, the peak-to-peak angular magnetoresistance is maximum and equal to the total TMR. Indeed, the antiparallel magnetic state is achieved at $\Phi=\pi$, even if the deviation $\Psi$ of the hard magnetization vector is not expected to be negligible, particularly when the field is oriented along the steps. The principle of the measurement is depicted in Fig. 4.

Measurements of the TMR angular variation in the highfield regime have been performed on junctions grown on $8^{\circ}$ misoriented $\mathrm{Si}$ substrates for $H=550$ Oe. Results are reported in Fig. 5 together with the ideal cosine shape repre-

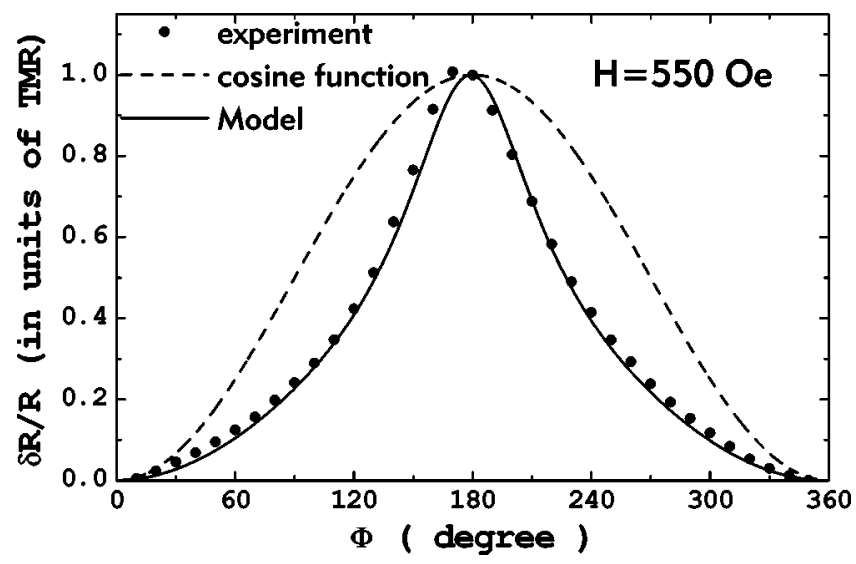

FIG. 5. High-field angular dependence of the TMR: experimental (circles) obtained for $H=550 \mathrm{Oe}$, ideal cosine response (dashed line), and theoretical response from relationships (19) and (20) corresponding to $\Gamma_{e x c}=1.9$ and $\delta_{d}=0.5$.
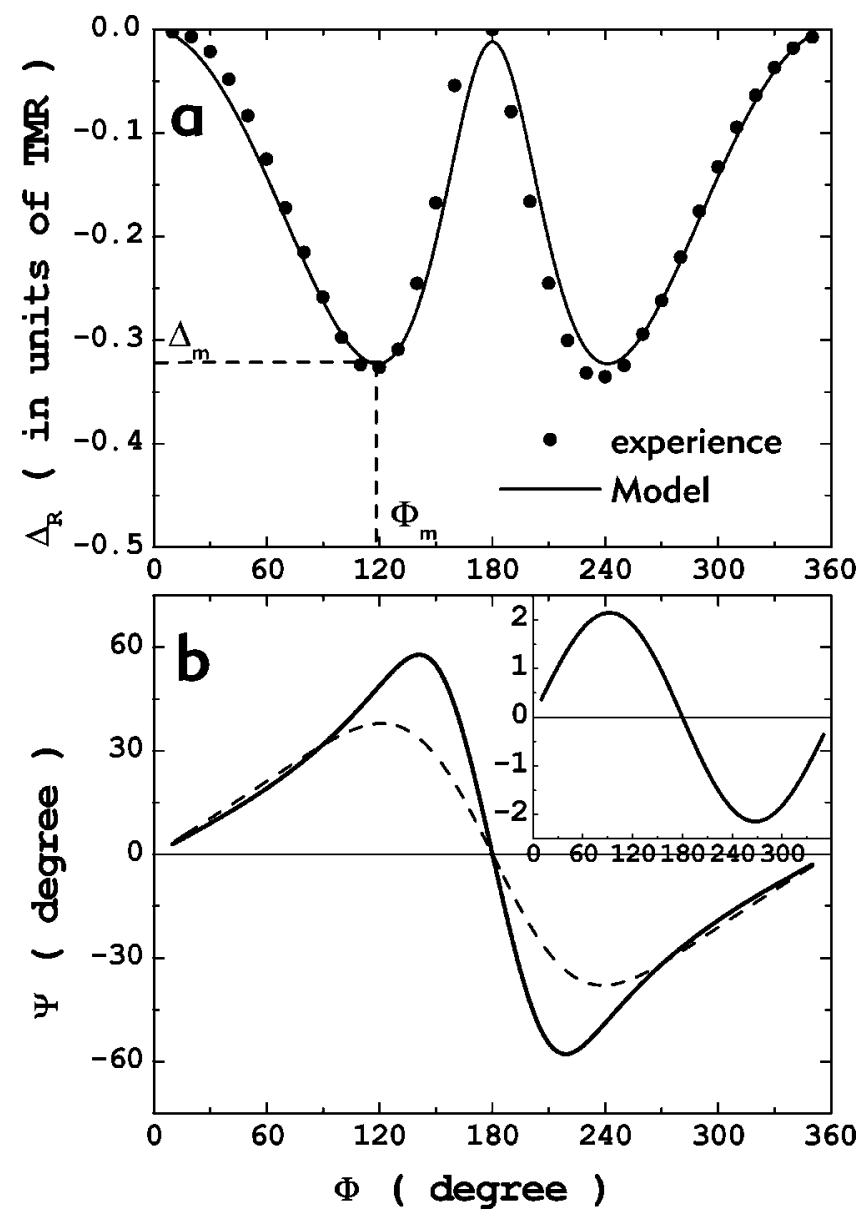

FIG. 6. (a) Deviation from the ideal cosine response: experimental (circles) and theoretical from formula (22) calculated with $\Gamma_{\text {exc }}=1.9$ and $\delta_{d}=0.5$. (b) plot of the angle deviation $\Psi$ of the exchange-biased magnet from its nominal pinning direction for $H$ $=550$ Oe with (straight line) and without (dashed curve) the dipolar field anisotropy contribution. In inset, the extrapolated deviation $\Psi$ (in degrees) expected for $h=0.4(H=40 \mathrm{Oe})$ is shown corresponding to results of Sec. III.

sented by the dashed line. The signal is reversible and has a total magnitude of $25 \%$ equal to the total TMR, as expected.

The deviation from the cosine function, called $\Delta_{R}$, is plotted in Fig. 6(a). It mainly originates from the behavior of the hard magnet under the influence of the field $H$ as well as the anisotropy of the interlayer coupling $H_{d}$.

\section{B. Model}

\section{Effects of the unidirectional exchange coupling}

When the unidirectional exchange anisotropy is not largely stronger than the applied field $H$, the hard magnetization may deviate from its nominal position. The exchangebias energy contribution (per magnetization unit) of the hard ferromagnet can be written as: ${ }^{28}$

$$
\epsilon_{e x c}=-H_{e x c} \cos \psi-\frac{K_{e x c}}{2} \cos ^{2} \psi
$$


where $\psi$ is the angle between the magnetization vector and the nominal pinning direction, $H_{e x c}$ is the interfacial unidirectional bias whereas $K_{e x c}$ is the uniaxial contribution (in units of magnetic field) induced during/by the field cooling. If one considers deviation angles not too large, one can write

$$
\sin \psi=\frac{H \sin \Phi}{H_{e x c}+K_{e x c}+H \cos \Phi}
$$

or

$$
\sin \psi=\frac{\sin \Phi}{\Gamma_{e x c}+\cos \Phi}
$$

where $\Gamma_{e x c}=\left(H_{e x c}+K_{e x c}\right) / H$ can be viewed as the effective exchange force limiting the rotation of the biased layer. Note that this value $\Gamma_{e x c}$ can be estimated as the ratio between the larger coercive field, measured on the $R(H)$ curve at low temperature, and the external field $\Gamma_{e x c}=H_{C}^{-} / H$.

\section{Effects of the dipolar field anisotropy}

A second source of distortion is introduced by unidirectional modulated structures played by step-bunched misoriented Si substrates. They present an artificial roughness that acts as an anisotropic "orange-peel" coupling. ${ }^{33}$ Indeed, one can separate the dipolar field $H_{d}$ into isotropic $H_{d}^{\|}$and anisotropic $H_{d}^{\perp}-H_{d}^{\|}$contributions induced by the steps. This is more pronounced for large misorientation $\left(\alpha=8^{\circ}\right)$ and was shown to vanish in the case of $\alpha=4^{\circ}$.

If $\Phi$ and $\psi$ locates the two magnetizations with respect to the direction perpendicular to the steps, the interlayer dipolar energy can be put in the following form:

$$
\epsilon_{d}=H_{d}^{\|} \sin \Phi \sin \psi+H_{d}^{\perp} \cos \Phi \cos \psi
$$

In the previous section, the intrinsic contribution was measured to be close to $H_{d}^{\|} \simeq 8$ Oe (for $\alpha=4^{\circ}$ ) and will be neglected in the following. Nevertheless, the contribution

$$
\epsilon_{d}=-H_{d}^{\perp} \cos \Phi \cos \psi
$$

remains and must be added to the total energy leading to

$$
\sin \psi=\frac{\sin \Phi}{\Gamma_{e x c}+\left(1+\frac{H_{d}^{\perp}}{H}\right) \cos \Phi}=\frac{\sin \Phi}{\Gamma_{e x c}+\left(1+\delta_{d}\right) \cos \Phi}
$$

with $\delta_{d}=H_{d}^{\perp} / H$.

\section{Angular magnetoresistance}

The measured angular dependence of the magnetoresistance can be evaluated through the following relationship:

$$
\frac{\delta R}{R} \simeq-\frac{\mathrm{TMR}}{2} \cos (\Phi-\psi) \simeq-\frac{\mathrm{TMR}}{2}(\cos \Phi+\sin \Phi \sin \psi)
$$

and the deviation from the ideal cosine response reads

$$
\Delta_{R} \simeq-\frac{\mathrm{TMR}}{2} \sin \Phi \sin \psi .
$$

From Eq. (16), one obtains

$$
\Delta_{R} \simeq-\frac{\mathrm{TMR}}{2} \frac{\sin ^{2} \Phi}{\Gamma_{e x c}+\left(1+\delta_{d}\right) \cos \Phi} .
$$

The deviation of the angular magnetoresistance from the ideal cosine function is driven by two parameters $\Gamma_{e x c}$ and $\delta_{d}$ that can be extracted experimentally by pointing the angle $\Phi_{m}$ corresponding to the minimum value $\Delta_{m}$ of $\Delta_{R}$ in units of TMR [Fig. 6(a)]. The differentiation of $\Delta_{R}$ with respect to $\Phi$ yields

$$
\Gamma_{e x c}=\frac{1+\cos ^{2} \Phi_{m}}{2 \Delta_{m}}
$$

and

$$
1+\delta_{d}=-\frac{\cos \Phi_{m}}{\Delta_{m}}
$$

Experimental values, $\cos \Phi_{m}=-0.4848$ and $\Delta_{m}=0.325$, give $\Gamma_{e x c} \simeq 1.9$ and $\delta_{d} \simeq 0.5$ together with $H_{e x c}+K_{e x c}$ $\simeq 1050 \mathrm{Oe}$ and $H_{d}^{\perp} \simeq 275$ Oe (for $H=550 \mathrm{Oe}$ ) in agreement with values deduced from $R(H)$ curves. ${ }^{30}$ Angular measurements are then well reproduced without use of any free parameters [Figs. 5 and 6(a)].

\section{Analysis of the angle deviation $\Psi$}

The angle deviation $\Psi$ of the hard magnetization given by the expression (19) is plotted as a straight line in Fig. 6(b) considering the parameters $\Gamma_{e x c}$ and $H_{d}^{\perp}$ quoted above. The maximum $\Psi$ value estimated at about $60^{\circ}$ is far from negligible and originates by part from the anisotropic dipolar effects. The own contribution of the loss of the exchange anisotropy is represented by the dashed curve maximum at $\Phi_{\max } \simeq 40^{\circ}$. As we can note, the deviation $\Psi$ is quite large for this range of field, which discards the possibility of a fine analysis of the angular dependence of the magnetoresistance.

One can extend the present model to estimate the angle deviation occurring in the low-field regime for $h=0.4$ (40 Oe) corresponding to $\Gamma_{\text {exc }}=26.6$ and $\delta_{d}=0\left(H_{d}^{\perp}=H_{d}^{\|}\right)$. The result plotted on the inset of Fig. 6(b) presents a maximum angle deviation approaching $2^{\circ}$, which introduces a smaller correction than the dipolar effects and can be neglected as it was done.

\section{CONCLUSION}

In conclusion, we have reported accurate angular measurements of the tunnel magnetoresistance supplied by crossed-biased magnetic junctions for various ranges of field. We have taken advantage of the low-field susceptibility property of the perpendicular magnetic configuration to investigate the angular variation of the tunnel conductivity by controlling the orientation of the soft-layer magnetization continuously. By performing phase analysis, we have pro- 
vided the experimental evidence that the conductivity depends linearly on the cosine of the angle made by the two magnetizations, as expected from the Slonczewski model. This leads in parallel to a more complex variation of the resistance and consequently, a gain in the signal linearity is expected by measuring the tunnel conductance.

The more intuitive geometry performed at higher field saturating the sensitive layer is shown to be more critical because it forces the rotation of the hard-layer magnetization, which discards the opportunity to extract the intrinsic angular response of the TMR in this regime. However, high-field measurements are shown to be a valuable method to study the magnetic properties of magnetic layers grown for spinelectronics tunnel devices.

\section{ACKNOWLEDGMENTS}

This work has been partially supported by the European Commission through Grants Nos. BR CT98-0657 ("Tunnelsense") and E-32464 ("Massdots"). One of us (J.B.) acknowledges the support of the European Commission through Grant No. ERBFMRX-CT97-0124 ("Dynaspin").
${ }^{1}$ J. Moodera, L. Kinder, T. Wong, and R. Meservey, Phys. Rev. Lett. 74, 3273 (1995).

${ }^{2}$ T. Miyazaki and N. Tezuka, J. Magn. Magn. Mater. 139, L231 (1995).

${ }^{3}$ Throughout this paper, we have defined the so-called TMR by the maximum difference of the conductivity $G_{p}-G_{a p}$ normalized by its averaged value $\bar{G}=\left(G_{p}+G_{a p}\right) / 2 . \quad \mathrm{TMR}=2\left(G_{p}\right.$ $\left.-G_{a p}\right) /\left(G_{p}+G_{a p}\right)=2\left(R_{a p}-R_{p}\right) /\left(R_{p}+R_{a p}\right)$.

${ }^{4}$ M. Juillère, Phys. Lett. 54A, 225 (1975).

${ }^{5}$ Y. Lu, R.A. Altman, S.A. Rishton, P.L. Trouilloud, G. Xiao, W.J. Gallagher, and S.S.P. Parkin, Appl. Phys. Lett. 70, 2610 (1997).

${ }^{6}$ M. Tondra, J.M. Daughton, D. Wang, R.S. Beech, and A. Fink, J. Appl. Phys. 83, 6688 (1998).

${ }^{7}$ M. Tondra, J.M. Daughton, C. Nordman, D. Wang, and J. Taylor, J. Appl. Phys. 87, 4679 (2000).

${ }^{8}$ D. Lacour et al. (unpublished).

${ }^{9}$ S. Gider, B. Runge, A. Marley, and S. Parkin, Science 281, 797 (1998).

${ }^{10}$ S.S.S.P. Parkin K.P. Roche, M.G. Samant, P.M. Rice, R.B, Beyers, R.E. Scheuerlein, E.J. O'Sullivan, S.L. Brown, J. Bucchigano, D.W. Abraham, Y. Lu, M. Rooks, P.L. Trouilloud, R.A. Wanner, and W.J. Gallagher, J. Appl. Phys. 85, 5828 (1999).

${ }^{11}$ D. Wang, M. Tondra, J.M. Daughton, C. Nordman, and A.V. Pohm, J. Appl. Phys. 85, 5255 (1999).

${ }^{12}$ L.B. Steren, A. Barthelemy, J.L. Duvail, A. Fert, R. Morel, F. Petroff, P. Holody, R. Loloee, and P.A. Schroeder, Phys. Rev. B 51, 292 (1995); T. Valet and A. Fert, ibid. 48, 7099 (1993).

${ }^{13}$ P. Dauguet, P. Gandit, J. Chaussy, S.F. Lee, A. Fert, and P. Holody, Phys. Rev. B 54, 1083 (1996).

${ }^{14}$ J. Slonczewski, Phys. Rev. B 39, 6995 (1989).

${ }^{15}$ R. Landauer, IBM J. Res. Dev. 1, 223 (1957).

${ }^{16}$ D. Ferry and S. Goodnick, Transport in Nanostructures (Cambridge University Press, Cambridge, England, 1997).

${ }^{17}$ The resistance is generally assumed to vary like $R=R_{\perp}[1$ - (TMR/2) cos $\Theta$ ] where $R_{\perp}$ is the resistance measured in the perpendicular magnetic configuration.

${ }^{18}$ See, for example, J. Moodera and L.R. Kinder, J. Appl. Phys. 79, 4724 (1996).

${ }^{19}$ M. Sussiau, F. Nguyen Van Dau, P. Galtier, A. Encinas, and A. Schuhl, J. Magn. Magn. Mater. 165, 1 (1996).

${ }^{20}$ The analogy with magneto-optics is obvious. The differential Kerr effect, which is turned into account to detect a slight change of the magnetization vector [see, for example, $\mathrm{K}$. Postava et al., J. Magn. Magn. Mater. 163, 8 (1996)] can be viewed, inversely, as an elegant method to modulate the light intensity by acting on the magnetization orientation.

${ }^{21}$ A. Anguelouch, B.D. Schrag, G. Xiao, Y. Lu, P.L. Trouilloud, R.A. Wanner, W.J. Gallagher, and S.S.P. Parkin, Appl. Phys. Lett. 76, 622 (2000).

${ }^{22}$ R.C. Sousa, J.J. Sun, V. Soares, P.P. Freitas, A. Kling, M.F. da Silva, and J.C. Soares, Appl. Phys. Lett. 73, 3288 (1998).

${ }^{23}$ J.J. Sun, K. Shimazawa, N. Kasahara, K. Sato, S. Saruki, T. Kagami, O. Redon, S. Araki, H. Morita, and M. Matsuzaki, Appl. Phys. Lett. 76, 2424 (2000).

${ }^{24}$ M. Sussiau, F.N.V. Dau, P. Galtier, and A. Schuhl, Appl. Phys. Lett. 69, 857 (1996).

${ }^{25}$ H. Jeong and E. Williams, Surf. Sci. Rep. 34, 171 (1999).

${ }^{26}$ W. Meiklejohn and C. Bean, Phys. Rev. 102, 1413 (1956).

${ }^{27}$ W. Meiklejohn and C. Bean, Phys. Rev. 105, 904 (1957).

${ }^{28}$ See, for example, the review article J. Nogués and I. Schuller, J. Magn. Magn. Mater. 192, 203 (1998).

${ }^{29}$ B. Dieny, V.S. Speriosu, S.S.P. Parkin, B.A. Gurney, D.R. Wilhoit, and D. Mauri, Phys. Rev. B 43, 1297 (1991).

${ }^{30}$ At $30 \mathrm{~K}$, we have measured on $\mathrm{CoO}$ a unidirectional exchange bias $H_{e x c}$ and uniaxial exchange anisotropy $K_{e x c}$, respectively, equal to 250 and 800 Oe whereas its Néel temperature was estimated at about $180 \mathrm{~K}$.

${ }^{31}$ F. Montaigne, J. Nassar, A. Vaurès, F. Nguyen Van Dau, F. Petroff, A. Schuhl, and A. Fert, Appl. Phys. Lett. 73, 2829 (1998).

${ }^{32}$ W. Brinkman, R. Dynes, and J. Rowell, J. Appl. Phys. 41, 1915 (1970).

${ }^{33}$ L. Néel, C.R. Hebd. Seances Acad. Sci. 255, 1545 (1962).

${ }^{34}$ This expression is rigorous at the order considered and can be derived by differentiating the energy derivative of the magnetization in the vicinity of the equilibrium state $\theta_{0}$ corresponding to $H=0$ and given by $\cos \theta_{0}=H_{d} / H_{K}^{\text {Step }}$. At the upper order, the angle modulation $\delta \theta$ is calculated to be $[-2 h \cos (\Phi+\phi)] /[1$ $\left.+h \sin (\Phi+\phi)+\sqrt{\{1+h \sin (\Phi+\phi)\}^{2}+2 h^{2} \cos ^{2}(\Phi+\phi)}\right]$, which does not introduce any relevant corrections.

${ }^{35}$ Expressions of $\delta R / R_{\perp}$ can be put into a general form as $[(\mathrm{TMR} / 2) h \cos (\Phi+\phi)] /\left[1+h \sqrt{1+(\mathrm{TMR})^{2} / 4} \sin (\Phi+\phi+\delta)\right]$ where $\delta$ is a small phase equaling TMR/ $\left[2 \times \sqrt{1+(\mathrm{TMR})^{2} / 4}\right]$ (0) in the case of a linear response of the conductivity (resistance) vs $\cos \theta$.

${ }^{36}$ This integral is not straightforward to calculate. However, it approaches $(\mathrm{TMR} h / 2)^{2} \int \cos ^{2} \Phi d \Phi /(1+h \sin \Phi)^{2} \quad$ or $(\mathrm{TMR} / 2)^{2} h \int \sin \Phi d \Phi /(1+h \sin \Phi)$, which can be easily developed in order of $h$. 